\title{
Marie Ebner-Eschenbach und das Judentum
}

\author{
Ingeborg Fialová
}

\begin{abstract}
Der Aufsatz wird sich mit dem oben angegebenen Thema befassen, trotzdem dazu freilich bereits Einiges gesagt worden ist ${ }^{1}$ - ich selbst schrieb vor Jahren eine Studie, in der ich die jüdischen Figuren bei Marie Ebner-Eschenbach mit denen bei Ferdinand von Saar und Jakob Julius David vergleiche ${ }^{2}$. Diese Studie wird hier hin und wieder zitiert.

Von der Ebner weiß man über ihre Einstellung zum Judentum - vor allem aus ihren Tagebüchern und Briefen - dass sie die Äußerungen des modernen Antisemitismus leidenschaftlich hasste, sie für gesellschaftliche Grobheit, für einen Durchbruch der Pöbelhaftigkeit hielt, dermaßen, dass sie dem von Bertha von Suttner gegründeten „Verein zur Abwehr des Antisemitismus“ (gegründet 1891) - zusammen mit ihrem Mann Moritz von Eschenbach - sofort beitrat ${ }^{3}$ und sich häufig über das neu-antisemitische Wien abschätzig äußerte, ihm die patriarchalisch-liberale Ruhe ihres geliebten Heimatortes Zdislawitz vorzog:
\end{abstract}

„Die Sonne scheint hell über dem antisemitischen Wien... ${ }^{4}$ Nur fort aus Wien! Nur fort aus dieser von der antisemitischen Krätze ergriffenen Stadt.... Die Straßen schimmern in antise-

1 Vgl. z.B. den Aufsatz von Egon Schwarz: Jüdische Gestalten bei Marie Ebner-Eschenbach und Ferdinand von Saar. In: The german quarterly, 63, 2, 1990, S. 173-186, den ich hauptsächlich als Folie für meine Ausführungen benutze.

2 Jüdische Figuren und das Thema der jüdischen Assimilation bei Marie von Ebner-Eschenbach, Ferdinand von Saar und Jakob Julius David. In: Spurensuche in Sprach- und Geschichtslandschaften. Festschrift f. Ernst Erich Metzner. LIT-Verlag, Münster/Hamburg/London, 2003, S. 123-134. Im Netz einsehbar auf dem Portal Kakanienrevisited.

3 Vgl. Brigitte Hamann, »Der Verein zur Abwehr des Antisemitismus«, in: Jüdisches Museum der Stadt Wien (Hrsg.), Die Macht der Bilder. Wien 1995.

4 Handschriftlich geschriebenes Tagebuch (im folgenden TB), aufbewahrt im Brünner Staatsarchiv. TB 8.1.1898. Vgl. dazu: Jiř́ Veselý: Marie von Ebner-Eschenbach und die anderen. In: Deutschböhmische Literatur, Univerzita Palackého, Olmütz 2001, S. 65-84

5 TB 16. 5. 1899. 
mitischen Schmutz... ${ }^{6}$ Moritz angekommen. „In Zdislawitz ist es schöner als in Wien.“ O wie hat er recht."7

Die ,jüdischen Themen“ sind bei der Ebner (und bei keinem der drei genannten mährischen Autoren) nicht zentral, doch trotz der Marginalität der jüdischen Thematik treffen alle drei Autoren zielsicher die - zu ihrer Zeit - zentralste „jüdischen Frage“, die Problematik der jüdischen Assimilation, das sie belletristisch anhand einiger jüdischer Figuren in ihrem Werk behandeln. Ebner-Eschenbachs Gelingen bei der Formung und Abbildung jüdischer Figuren wird sogar auf Wikipedia-Seiten festgehalten, allerdings recht negativ eingeschätzt:

„Ihr ganzes Leben lang kämpfte sie gegen die etablierten Gedanken ihrer Zeit. Sie schrieb in der Überzeugung, ihre Schriften könnten die Gedanken ihrer Zeit verändern. Ihre Absicht war, Sittlichkeit und Humanismus zu vermitteln. Obwohl Ebner-Eschenbach Mitgründerin eines Vereins zur Bekämpfung des Antisemitismus war, konnte sie sich von entsprechenden Klischees bei der Beschreibung jüdischer Nebenfiguren in ihren Werken nicht völlig befreien." ${ }^{8}$

Dieser Diskrepanz - öffentliches Engagement gegen den Antisemitismus einerseits und klischeehafte (sprich antisemitisch gefärbte) belletristische Bearbeitung jüdischer Themen/Figuren andererseits - will ich im Folgenden nachgehen:

Auf welche Nebenfiguren bezieht sich denn überhaupt die negative Einschätzung auf Wikipedia-Seiten (denn explizit gesagt wird es nicht)? Doch nicht auf die Nebenfigur des jungen jüdischen Hausierers Salomon Levi aus der Erzählung Der Vorzugsschüler (aus dem Jahre 1901), der zwar mit typischen äußeren Zeichen eines armen, in Wien gestrandeten Ostjuden (also einer typische Zielscheibe für damals modische, karikaturhafte, abgeschmackte antisemitische Witzeleien) ausgestattet wird:

„...Salomon Levi war fünfzehn Jahre alt und trug schiefgetragene Stiefel, einen schwarzen Kaftan, einen steifen breitkrempigen Hut. Seine eingefallenen Wangen entlang baumelten ein paar glänzende rabenschwarze Schläfenlocken,“9

doch im Ganzen im mitleidvollen Blick der Erzählerin als positive, weil bedauernswerte, leidbeladene Figur erscheint, gleichsam ein neuer Ahasver, den außerdem sein Bildungsdurst auszeichnet: „Mir möchte leicht sein das Studieren“ - so im nachgeahmten Judendeutsch der Figurenrede oder weiter im klagelidartigen Kommentar der Erzählerin:

„Auch der war elend, dem es Seligkeit gewesen wäre, in der Schule zu sitzen [...] und der auf und ab wandeln musste vom frühen Morgen bis in die späte Nacht im Staub und Sonnebrand,

6 TB 27. 2. 1898.

7 TB 31. 10. 1882.

8 https://de.wikipedia.org/wiki/Marie_von_Ebner-Eschenbach

9 Marie von Ebner-Eschenbach: Gesammelte Werke in drei Bänden. Bd. 1: Das Gemeindekind. Novellen, Aphorismen, München 1956-1958, S. 521. 
und sah so krank aus, und seine schmächtige Gestalt war schon ganz schief vom Tragen des schweren Warenkastens." 10

Salomon Levi ist auch der Mensch (mit Worten Eugen Schwarz'),

\begin{abstract}
„der den toten Freund am tiefsten beklagt, so sehr, dass er darüber, entgegen der populären Vorstellungen vom jüdischen Geist und Geschäftsgeist, seines Besitztums und seiner Waren, die schließlich seinen Lebensunterhalt ausmachen, nicht achtet...“ und „[die Ebner] übernimmt das gängige Judenbild, humanisiert es aber psychisch und widerlegt die Klischees."11
\end{abstract}

Dann also ein zweiter Versuch: Nathanael Rosenzweig, der Doktor und Kreisphysikus aus Ebners gleichnamiger Erzählung von 1882: Seiner Figur versuchte ich in meinem erwähnten Aufsatz näher zu kommen, indem ich sie mit der Figur des Medizinstudenten Simon Siebenschein aus Davids Roman Am Wege sterben von 1900 verglich. Die Inhalte der beiden Erzählungen sind schnell erzählt:

Nathanael Rosenzweig, der Doktor und Kreisphysikus, der in Ebners Erzählung im Galizischen um 1845 agiert, wird Zeuge der Vorbereitungen eines revolutionären Aufstandes - und trotzdem er anfänglich von der revolutionären Begeisterung des polnischen Adels nicht viel hält und vor dem revolutionären Aufwachen der Bauern eher Angst hat, lässt er sich durch überzeugende Kraft der prophetischen Reden des „Sendboten" Dembowski hinreißen, wird zum Treuen Anhänger der christlichen und sozialen revolutionären Gedanken - und bleibt es, auch nachdem der eigentliche Aufstand niedergemetzelt wurde. ${ }^{12}$

Davids Roman zeigt kalleidoskopartig die Schicksale einzelner Studenten aus einer lockeren Tafelrunde in Wien um 1885, die alle nicht fähig sind, in der Großstadt Fuß zu fassen und an ihr zugrunde gehen, „am Wege sterben“. Der jüdische Medizin-Student Simon Siebenschein ist die einzige Ausnahme: Trotz der erschütternden Erfahrung einer tragischen Liebe, da wegen seiner Unfähigkeit, Gefühle zu äußern, ein unschuldiges Mädchen starb, und trotz seiner allgemeinen Werte-Skepsis, schafft er den Sprung ins Berufsleben und wird Armenarzt.

Nathanael Rosenzweig und Simon Siebenschein sind sehr ähnliche Figuren, sie weisen dermaßen erstaunliche Parallelen auf, das man tatsächlich versucht ist, von Klischees in der Darstellung jüdischer Figuren zu sprechen:

Beide sind Mediziner, beide ergriffen ihren Beruf nicht etwa, weil ihnen keine andere intellektuelle Laufbahn offen gestanden hätte (wie es in der Zeit Nathanael Rosenzweigs noch tatsächlich der Fall war, nicht mehr aber in der Zeit Simon Siebenscheins), sondern, weil ihnen

10 Ebd. 551.

11 Schwarz, 176.

12 Den historischen Kontext des polnischen Aufstandes beschreibt Wojciech Kunicki: Irredenta uns Statsräson. Gestaltung des Raumes in den Erzählungen Jakob Szela und Der Kreisphysikus in: Karl Konrad Polheim (Hrsg.): Marie von Ebner-Eschenbach. Ein Bonner Symposion zu ihrem 75. Todesjahr. Peter Lang, Bern u. a. 1994, S. 209-228. 
dieser Beruf gesellschaftliche Geltung und Besitz zu bescheren versprach. Von Nathanael Rosenzweig erfährt man gleich auf der ersten Seite der Novelle, dass „...Erwerben der Inbegriff all seines Dichtens und Trachtens [war], Geld erwerben, Kenntnisse, Gunst [...], erwerben und ja nichts umsonst hergeben." (EE245) ${ }^{13}$ Vom Studenten Siebenschein heißt es - weniger explizit - dass er ein „rastloser Lerner“ war, der sich von seinen verbummelten Studentenkollegen gewaltig unterscheid, da er weder trank, noch Schulden machte, sondern zielgerichtet auf seine künftige Laufbahn hinarbeitete, denn ,jeder Tag, den er unnütz vertat, zwang ihn, sich zu bescheiden“ $(\mathrm{D} 57)^{14}$.

Diese ausschließliche Ausrichtung aufs Ziel hin macht die Helden hartherzig und hochmütig. Rosenzweig verbietet sich rigoros jede „mitleidige Regung“, jede „hemmende Rücksicht“ (EE245). Siebenschein wird gleich in seiner Erstaufnahme durch den Erzähler als „schroffer, schweigsamer [...] hochmütiger und nervöser Mensch“ (D32) dargestellt, der sich mit den niederen Schichten nicht einlassen möchte und lieber allein sitzt, „eher er sich unter das Volk mischte“ (D57). Diese Eingangscharakteristik macht die jüdischen Figuren dem Leser freilich einigermaßen unsympathisch, trotzdem sich beide Erzähler bemühen, diesen Eindruck etwas zu entschärfen: Die Ebner mit dem Hinweis auf die entbehrungsreiche Jugend Nathanael Rosenzweigs am Rande des Hungertodes, die eben als Motivation für sein heutiges Verhalten gilt, David durch die Einpflanzung Simon Siebenscheins in die Gruppe verbummelter Wiener Studenten, die allesamt verkrachte Existenzen mit gewaltigen Charakterschwächen sind und für den Leser sowieso unsympathisch. Trotzdem fühlt man sich als Leser des 21. Jahrhunderts unangenehm an das antisemitische Klischee des „rastlosen Juden“, des „modernen Ahasvers“, erinnert. Dieses Leser-Gefühl wird durch die häufige explizite Wiederholung des Adjektivs „rastlos“ gesteigert (bei beiden Autoren in verschiedenen Verbindungen), und durch die äußere Charakteristik der Helden verstärkt, denn beide sind keine Schönheiten.

Der Makel der körperlichen Gebrechlichkeit begleitete Nathanael Rosenzweigs Jugend, trotzdem sich heute „seine kreuzspinnenartigen Extremitäten“ zu „muskulösen Armen und Beinen kräftigten“ (EE245). In dieser physischen Entwicklung des Helden von einer „dürftigen Leiblichkeit, die in den gewöhnlichen antisemitischen Werken den Juden anhaftet [...] zum Reckenhaften [...] zur verbalen Annäherung der Körperlichkeit des jüdischen Doktors an die Lichtgestalten der germanischen Sagen,“ sieht Eugen Schwarz bereits den Beweis für Ebners „Widerlegung der Klischees. "15 Simon Siebenschein wird als „lang und hager“ beschrieben, als „alt und unfertig zugleich“ mit einem Gesicht, „von einer kränklichen bräunlich-blassen Färbung, als hätte man durch Milch einen starken Rauch gehen lassen“ (D43). David verknüpft diese Beschreibung Simon Siebenscheins mit seinen ,jüdischen Rassenanlagen“: „...denn etwas vom Hochmute seiner Rasse war in ihm“ (D57), „die Nervosität seiner Rasse steigerte seine

13 Marie von Ebner-Eschenbach: Der Kreisphysikus. In: Erzählungen, autobiographische Schriften. WinklerVerlag, München, keine Jahresangabe, S. 245-305. Die Seitenangabe der zitierten Stelle erfolgt direkt im Text. 14 Jakob Julius David: Am Wege sterben. Schuster und Loeffler, Berlin und Leipzig, 1900. Die Seitenangabe der zitierten Stelle erfolgt direkt im Text.

15 Schwarz $176 f$. 
Empfindlichkeit“ (D123), „die ganze Leidenschaftlichkeit seiner Rasse zuckte in ihm“ (D236) usw. Bei der Ebner - 18 Jahre früher - findet man logischer Weise noch kein, der modernen Rassenlehre entsprungenes, Vokabular, sondern Rosenzweigs anfänglich negative Charaktereigenschaften werden weniger im Jüdischen als vielmehr im allgemein Märchenhaft-Orientalischen verankert: „Mahnst du auch mich einmal, nie angetretenes Vätererbe - morgenländische Phantasie?“ (EE248) fragt Rosenzweig lächelnd, wenn er sich beim träumenden Bauen von Luftschlössern seiner Besitztümer ertappt.

Rosenzweigs und Siebenscheins Rastlosigkeit, Ehrgeizigkeit und Geizigkeit, Hochmütigkeit und Hartherzigkeit äußern sich freilich am deutlichsten in ihrer Beziehung zur menschlichen Umgebung: Während Nathanael Rosenzweigs hochmütig ironischer Abstand zum revolutionsbegeisterten polnischen Adel - in einer von der Ebner überaus witzig-ironisch gestalteten Szene - noch sympathisch wirkt,

\begin{abstract}
„,Der Augenblick, das fremde Joch abzuschütteln, ist gekommen... Das Zeichen zum Ausbruch der Revolution wird in Lemberg auf dem ersten Balle des Erzherzogs gegeben werden.' [...] ,Ich verstehe, auf jenem Revolutionsballe!' Ja, Doktor! Ja!' rief Gräfin Aniela dazwischen, ,dem Balle, auf dem wir ein welthistorisches Ereingis inaugurieren!' ,Bei der Mazurka oder bei der Francaise?’ ,Beim Kotillon...'“ (EE260f.)
\end{abstract}

und Rosenzweigs gut motivierter Verrat an den adeligen Aufständischen noch verständlich ist, stimmt sein offen getragener Hass gegenüber Armen, Bettlern und sonst Unbedarften schon nachdenklich, vor allem weil er gegen die positive Gestalt des jungen Schützlings Josef gerichtet ist. Einerseits erwies der Doktor seine Barmherzigkeit dadurch, dass er den Findling „vor vier Jahren von der Straße in einer eiskalten, herrlichen Winternacht aufgelesen hat" (EE249), andererseits äußert sich seine Hartherzigkeit in der Behandlung des Jungen, „seines Dieners, seines Hundes“ (EE249), den er geringschätzt, weil er „aus Büchern nicht zu lernen vermochte. Mit achtzehn Jahren noch las er nicht ohne Schwierigkeiten die einfachsten Kindergeschichten“ (EE252), und den er eigentlich aus tiefstem Herzen hasst, da er den Gedanken nicht loswerden kann, „dass der Findling seines Herren Brot umsonst oder doch fast umsonst esse“. (EE249) Und doch ist gerade Josef Träger des spontanen, guten, aufopfernden Menschentums, dem Ebner-Eschenbach in mehreren Erzählungen, meist in den Gestalten von Bauern, bäurischen Mädchen, Müttern und sogar Hunden ein Denkmal baute: Er ist seinem schroffen Herren treu, er ist genügsam, überaus fleißig, immer freundlich, menschlicher Gefühle und Regungen fähig - in allem ein Gegenbild, ein Korrektiv Rosenzweigs.

Denselben Typus stellt auch Jakob Julius David seinem Helden Simon Siebenschein gegenüber und lässt ihn an der Beziehung zum Mädchen Resi genauso scheitern wie Ebner ihren Rosenzweig in der Beziehung zu Josef seine Charaktermängel äußern lässt. Siebenschein bewundert von Weitem die aufopfernde Liebe, die die arme kleine Resi ihrem hinsiechenden Spielkameraden entgegen bringt, die stille Demut, mit der sie Quälerein der Mutter und des Untermieters aushält, deutet Resis Wesen richtig als Aufblitzen der Heiligkeit im Menschlichen, lässt jedoch keine Gefühlsregung - die er nämlich (ähnlich wie Rosezweig) als Schwäche empfindet - in sein verhärtetes Inneres 
hinein. Siebenschein lässt die kleine, der Schlechtigkeit der Welt nicht gewachsene Resi im Stich, lässt sie schließlich in den sicheren Tod laufen, wird an ihrem Tod mitschuldig.

Trotzdem werden beide Figuren, die am Anfang mehr oder weniger unsympathisch wirken, im Laufe der Geschichte zu positiven Helden: Nathanael Rosenzweig kehrt sich nicht ab von den christlich-sozialen Idealen des prophetischen Sendboten wie alle anderen, sondern lässt sich entzücken, ändert gründlich seine Weltanschauung und Lebenshaltung, wird mitleidsfähig und charitativ, erkennt plötzlich das Ausmaß der opferbereiten Treue Josefs an, beschenkt ihn mit seinem Gut (das ursprünglich das Ziel all seines Lebens und Trachtens war), versucht mit großzügigen Spenden das Leben der Bauern erträglicher zu machen und wird schließlich zum fahrenden Armenarzt. Als Lohn für diesen Umschwung empfängt er - im Epilog nach etwa 10 Jahren - die unerwartete Begegnung mit dem tot geglaubten Sendboten Eduard Dembowski. Die Erzählung endet idyllisch in der Umarmung der beiden und dem feierlichen Versprechen Rosenzweigs, ein treuer Jünger Dembowskis und seines Glaubens zu bleiben.

Simon Siebenschein findet nach dem Tode Resis trotz seines Zweifels an allen Werten des Lebens die Kraft, sein Studium zu Ende zu führen. Er zieht in eine Arbeitersiedlung und am Schluss des Romans - ebenfalls im Epilog - schließt er sich beim Ausflug ins Grüne symbolisch einer Gruppe junger sozialistischer Arbeiter an und wird ebenfalls zum Armenarzt.

Nur ein überempfindlicher Leser würde der Ebner und J. J. David die Ausnutzung antisemitischer Klischees vorwerfen, da sie doch in beiden Fällen - wie Egon Schwarz urteilt - nur übernommen werden, um widerlegt zu werden.

Zum viel massiveren Durchbruch antisemitischer Klischees kommt es allerdings bei der Präsentation von zwei Dorfjuden kurz vor dem Wendepunkt der Ebnerschen Novelle, als eine revolutionäre Versammlung in die Dorfkneipe des Juden Abraham einberufen wird. Man fühlt sich hier tatsächlich an die antisemitischen Kalender und Karrikaturen des 19. und 20. Jahrhunderts unangenehm erinnert, denn alle erdenklichen, die abgeschmacktesten und also wirksamsten „volkstümlichen“ antisemitischen Klischees werden hier benutzt. Die zwei Absätze lauten:

„,Schaff mit Platz, Abraham,' sprach der Doktor, ,ich bin’s, ich, Doktor Rosenzweig.' ,Gott der Gerechte!' stieß der Wirt erschrocken hervor, fasste sich aber gleich und patschte dienstwillig in den Sumpf [...] Ein Gibor! Schema Isroel, ein Gibor der gewaltige Doktor!' raunte Abraham einem missgestalteten Wesen zu, das plötzlich im Dunkel, geräuschlos wie eine Eidechse, krummbeinig wie ein Kobold, neben ihm aufgetaucht war. Es wiegte den unförmigen Kopf, seine nachtschwarzen Augen funkelten klug und feurig. ,Er ist eingezogen zu spionieren, Tateleben. Wir wollen ihm kommen zuvor, dass uns nicht kann begegnen ein Unglück,' flüsterte der Kleine [...] ,Ich will nehmen ein Pferd, Tateleben, und reiten nach Tarnow wie ein Windstoß, zu melden bei der Polizei, dass bei uns Versammlung halten die rebellischen Gojim und dass die kaiserliche Regierung soll ausschicken gegen sie Soldaten, wenn es is gefällig der kaiserlichen Regierung.' Abraham betrachtete seinen Sprössling mit Blicken bewundernder Liebe: , Reit wie ein Windstoß, mein Sohneleben, dass du mit Gott bald kommst ans Ziel. Reit,' 
wiederholte er, und er setzte in naiver Fürsorge hinzu: ,Tu dich nur nehmen in acht, dass du nicht kommst um deine geraden Glieder."

Das ist also wahrscheinlich die Stelle, die der Ebner auf Wikipedia-Seiten vorgeworfen wird. Eugen Schwarz beurteilt die zwei Figuren wie „einer jüdischen Ganovengalerie entlehnt [...] als kämen sie aus der Unterwelt eines Dickensschen Romans [...] als eine Szene, die vom Stürmer nicht ekliger hätte erfunden werden können,“ und „entschuldigt“ sie durch den Hinweis darauf, dass die Handlungsweise der beiden Dorfjuden tatsächlich der „geschichtlichen Lage entspricht, da den polnischen Juden an einer siegreichen polnischen Revolution nicht gelegen sein konnte und sie deshalb kaisertreu waren “. ${ }^{16}$

Dieser Befund „entschuldigt“ allerdings die Benutzung der antisemitischen Klischees nicht - hier also meine zwei Erklärungsangebote:

Erstens: Die Szene mit den beiden Dorfjuden ist dermaßen überzeichnet (v.a. der letzte Satz: ,Tu dich nur nehmen in acht, dass du nicht kommst um deine geraden Glieder.'“), und klingt wie ein Versatzstück aus einer Typenkomödie, dass man humoristische Absicht der Erzählerin vermuten kann, was man ja auch als eine „Umpolung“ gängiger antisemitischer Klischees deuten könnte. Die zweite Erklärung lehne ich an die Formulierung Hubert Lengauers an, die er in Bezug auf die Kontroverse zwischen Ferdinand Kürnberger und Ludwig August Frankl äußerte, welche Kontroverse sich auf das von Frankl geplante Schiller-Denkmal in Wien bezog und in antisemitisch gefärbten Attacken Kürnbergers auf Frankl gipfelten. Lengauer kommentiert:

\begin{abstract}
„Ist unser Sensorium für Antisemitismus in dem Maße gestiegen, dass wir in jeder einschlägigen Äußerung Auschwitz aufdämmern sehen? Dies einerseits zu Recht, denn es sind solche „zufälligen“ Äußerungen, die den Antisemitismus „salonfähig“ oder zu einer ignorierten Normalität gemacht haben, auf dessen Basis erst der Exzess der Schoa möglich war. Andererseits zu Unrecht, wenn man Autoren wie Kürnberger diesem Urteil unterwirft, denen - von anderen Grundlagen her - die Vorbereitung des Exzesses nicht grundsätzlich als Absicht unterstellt werden kann." 17
\end{abstract}

Diese Sätze können für die Ebner voll übernommen werden, da sie ihre beiden Erzählungen in derselben Zeit wie Kürnberger seine Attacken schrieb, in einer Zeit, da antisemitische „Schematisierung [...] ab den 1870er Jahren salonfähig und Teil der politischen Normalität wurden" 18 , da nach dem sog. Zeitalter des Liberalismus, der Phase einer relativ reibungslosen Emanzipation und Assimilation der Juden an die deutschösterreichische Kultur in Österreich (ähnlich wie in Deutschland) der sog. moderne Antisemitismus aufkam, der die mittelalterlichen religiösen antijüdischen Vorurteile mit dem neueren romantischen organisch-nationalistischen Gedankengut verknüpfte, sie

\footnotetext{
16 Schwarz 179.
}

17 Hubert Lengauer: Konkurrenz und Kompensation. L. A. Frankl und Ferdinand Kürneberger. In: Ludwig August Frankl (1810-1894). Eine jüdische Biographie zwischen Okzident und Orient. Hrsg. von Louise Hecht, Böhlau Verlag, Köln, Weimar, Wien, S. 138.

18 Lengauer 138. 
mit wirtschaftlicher Argumentation und schließlich mit rassistischen „Theorien“ anreicherte und diese alt-neue Weltanschauung in eine Sprach-Demagogie neuer, journalistischer Prägung einpackte. Vehikel des modernen Antisemitismus waren in Österreich (gleich wie in Deutschland) „gelehrte“ rassenantisemitische Schriften, „lustige“ Pamphlete und antisemitische Kalender für das Volk, Ritualmordprozesse, Verein- und Parteigründungen sowie Parteiprogramme. 1891 gründete Karl Lueger in Wien die offen antisemitische Christsoziale Partei, die ihn, ihren Gründer, 1897 auf die Höhe des Postens des Bürgermeisters von Wien erhob. In dieser gesellschaftlichen Situation, da die jüdische Assimilation trotz aller Anpassungsbemühungen der letzten Jahrzehnte durch den Ansturm des neuen Rassenantisemitismus ins Wanken gebracht wurde, schrieben die drei mährischen Autoren, im Zeitraum zwischen 1882 und 1900, dem neuralgischen Zeitraum der jüdischen Assimilation, ihre besprochenen Prosa-Texte.

Die Problematik der jüdischen Assimilation ist allerdings nicht die zentralste Idee der vorgestellten Texte - sie verfolgen jeweils eine andere Haupt-Idee - nur nebenbei sind die Helden als einzelne Projekte zur Lösung der Assimilationsfrage zu lesen und zu interpretieren: Nathanael Rosenzweig als einer, der aus dem strengen Individualismus des „sich auf sich selbst Verlassens“ in die wärmende Gemeinschaft der Welt- und Menschheitsverbesserer wechselte, Simon Siebenschein als eine zeitlich bedingte „Innovation“ Nathanael Rosenzweigs, da der Individualismus durch moderne (schopenhauersche und nietzschanische) Skepsis verschärft wurde und die (am Ende nur halbwegs erreichte) Gemeinschaft der Welt- und Menschheitsverbesserer bereits sozialistische Züge trägt.

Prof. PhDr. Ingeborg Fialová, Dr. / ingeborg.fialova@upol.cz

Univerzita Palackého, Filozofická fakulta, Katedra germanistiky Krrižkovského 10, 77180 Olomouc, CZ 